\title{
On the stability of delayed feedback controllers for discrete time systems
}

\author{
Ömer Morgül \\ Department of Electrical and Electronics Engineering, Bilkent University, 06533 Bilkent, Ankara, Turkey \\ Received 24 November 2003; received in revised form 17 May 2004; accepted 10 December 2004 \\ Available online 24 December 2004 \\ Communicated by A.P. Fordy
}

\begin{abstract}
We consider the stability of delayed feedback control (DFC) scheme for multi-dimensional discrete time systems. We first construct a map whose fixed points correspond to the periodic orbits of the uncontrolled system. Then the stability of the DFC is analyzed as the stability of the corresponding equilibrium point of the constructed map. For each periodic orbit, we construct a characteristic polynomial whose Schur stability corresponds to the stability of DFC scheme.

(c) 2005 Elsevier B.V. All rights reserved.
\end{abstract}

PACS: $05.45 . \mathrm{Gg}$

Keywords: Chaotic systems; Chaos control; Delayed feedback; Pyragas controller

\section{Introduction}

The study of various aspects of chaotic systems has received considerable interest in recent years. Among such aspects, the feedback control of chaotic systems is an important area due to its numerous potential applications. This subject has gained a great deal of attention after the seminal work of [1], where the term "controlling chaos" was introduced. The literature is quite rich on the subject, and for more information the interested reader may resort to, e.g., [2,3].

E-mail address: morgul@ee.bilkent.edu.tr (Ö. Morgül).
As in the classical feedback control theory, various objectives could be given for the chaotic systems, and depending on these objectives various control techniques could be used, see, e.g., [3]. One such control objective is the stabilization of unstable periodic orbits, [1]. Chaotic systems usually have many unstable periodic orbits embedded in their attractors. It was shown in [1] that some of these orbits may be stabilized by using small external input, hence it may be possible to obtain regular behaviour in such systems by using feedback control. The delayed feedback control (DFC), which is first proposed in [4], is one of such schemes and has received considerable attention due to its various attractive features. In this scheme, 
the feedback term is the multiplication of a gain with the difference between the current and one period delayed states. Therefore, if the system is already in the periodic orbit, this feedback term vanishes. The problem is to find an appropriate gain so that a given periodic orbit becomes stable.

Various applications of DFC has been reported in the literature, including electronic systems [5,6], lasers [7], magnetoelastic systems [8], traffic model [9], see also $[3,10]$ for more references. Although DFC is quite simple, its stability analysis is quite difficult, [10]. Several stability results has been obtained, see $[6,11-$ 13]. These results show that the DFC scheme has an inherent limitation, i.e., it cannot be applied for the stabilization of some periodic orbits, [11,12]. To overcome these limitations, various modifications have been proposed, see [14-20]. Recently a set of necessary and sufficient conditions to guarantee the stability of DFC for one-dimensional discrete time systems has been given in [13].

In this Letter we consider the stability of DFC scheme for (multi-dimensional) discrete time systems. To analyze the stability of DFC scheme, we use the approach given in [13]. We first construct a map whose fixed points correspond to the periodic orbits of the original system. Then the stability of the DFC scheme can be analyzed by studying the corresponding fixed points of this constructed map. To analyze the latter, we form the Jacobian of this map and then find its characteristic polynomial. The Schur stability of this polynomial can be used to study the stability of DFC scheme. In that respect, the results given in this Letter can be considered as a generalization of similar results given in [13] for one-dimensional case to multidimensional case.

This Letter is organized as follows. In Section 2 we introduce the notation and define the stability problem. In Section 3 we give our stability results. In the following section we present some simulation results. Finally we give some concluding remarks.

\section{Problem statement}

Let us consider the following discrete-time system

$x(k+1)=f(x(k))$, where $k=1,2 \ldots$ is the discrete time index, $x \in \mathbf{R}^{n}$, $f: \mathbf{R}^{n} \rightarrow \mathbf{R}^{n}$ is an appropriate function, which is assumed to be differentiable wherever required. We assume that the system given by (1) possesses a $T$ periodic orbit characterized by the set

$\Sigma_{T}=\left\{x_{1}^{*}, x_{2}^{*}, \ldots, x_{T}^{*}\right\}$,

i.e., for $x(1)=x_{1}^{*}$, the iterates of (1) yields $x(2)=$ $x_{2}^{*}, \ldots, x(T)=x_{T}^{*}, x(k)=x(k-T)$ for $k>T$.

Let $x(\cdot)$ be a solution of (1). To characterize the convergence of $x(\cdot)$ to $\Sigma_{T}$, we need a distance measure, which is defined as follows. For $x_{i}^{*}$, we will use circular notation, i.e., $x_{i}^{*}=x_{j}^{*}$ for $i=j(\bmod T)$. Let us define the following indices $(j=1, \ldots, T)$ :

$d_{k}(j)=\sqrt{\sum_{i=0}^{T-1}\left\|x(k+i)-x_{i+j}^{*}\right\|^{2}}$,

where $\|\cdot\|$ denotes any norm in $\mathbf{R}^{n}$. Without loss of generality, we will use standard Euclidean norm in the sequel. We then define the following distance measure

$d\left(x(k), \Sigma_{T}\right)=\min \left\{d_{k}(1), \ldots, d_{k}(T)\right\}$.

Clearly, if $x(1) \in \Sigma_{T}$, then $d\left(x(k), \Sigma_{T}\right)=0, \forall k$. Conversely if $d\left(x(k), \Sigma_{T}\right)=0$ for some $k_{0}$, then it remains 0 and $x(k) \in \Sigma_{T}$, for $k \geqslant k_{0}$. We will use $d\left(x(k), \Sigma_{T}\right)$ as a measure of convergence to the periodic solution given by $\Sigma_{T}$.

Let $x(\cdot)$ be a solution of (1) starting with $x(1)=x_{1}$. We say that $\Sigma_{T}$ is (locally) asymptotically stable if there exists an $\varepsilon>0$ such that for any $x(1) \in \mathbf{R}^{n}$ for which $d\left(x(1), \Sigma_{T}\right)<\varepsilon$ holds, we have $\lim _{k \rightarrow \infty} d\left(x(k), \Sigma_{T}\right)=0$. Moreover if this decay is exponential, i.e., the following holds for some $M \geqslant 1$ and $0<\rho<1(k>1)$ :

$d\left(x(k), \Sigma_{T}\right) \leqslant M \rho^{k} d\left(x(1), \Sigma_{T}\right)$,

then we say that $\Sigma_{T}$ is (locally) exponentially stable.

To stabilize the periodic orbits of (1), let us apply the following control law:

$x(k+1)=f(x(k))+u(k)$

where $u(\cdot)$ is the control input. In classical DFC, the following feedback law is used $(k>T)$ :

$u(k)=K(x(k)-x(k-T))$,

where $K \in \mathbf{R}^{n \times n}$ is a constant gain matrix to be determined. Now we state the stability problem as follows. 
Problem. Let $\Sigma_{T}$ be a period $T$ orbit of the system (1), and consider the system given by (6), (7). Find a gain matrix $K$ such that $\Sigma_{T}$ becomes stable.

\section{Stability analysis}

The stability analysis given here is similar to the one given in [13]. Let us consider the simple case $T=1$. In this case we have $\Sigma_{1}=\left\{x_{1}^{*}\right\}$ where $x_{1}^{*}=$ $f\left(x_{1}^{*}\right)$. In other words, period 1 orbits are the same as fixed points of $f$. By using change of variables $x_{1}(k)=x(k-1), x_{2}(k)=x(k)$, we can rewrite (6) and (7) as

$$
\begin{aligned}
& x_{1}(k+1)=x_{2}(k), \\
& x_{2}(k+1)=f\left(x_{2}(k)\right)+K\left(x_{2}(k)-x_{1}(k)\right) .
\end{aligned}
$$

Let us define $\hat{x}=\left(x_{1} x_{2}\right)^{T} \in \mathbf{R}^{2 n}$, where superscript $T$ denotes transpose, and define $F: \mathbf{R}^{2 n} \rightarrow \mathbf{R}^{2 n}$ as $F(\hat{x})=\left(Y_{1} Y_{2}\right)^{T}$ where $Y_{1}=x_{2}, Y_{2}=f\left(x_{2}\right)+K\left(x_{2}-\right.$ $\left.x_{1}\right)$. Hence, $(8)$ can be equivalently written as

$\hat{x}(k+1)=F(\hat{x}(k))$.

The fixed points of $F$, i.e., the solutions of $F\left(\hat{x}^{*}\right)=$ $\hat{x}^{*}$, are given as $x_{1}^{*}=x_{2}^{*}=f\left(x_{1}^{*}\right)$. Hence, for any period 1 orbit $\Sigma_{1}=\left\{x_{1}^{*}\right\}$ of (1), there exists a fixed point of $F$ of the form $\hat{x}^{*}=\left(x_{1}^{*} x_{1}^{*}\right)^{T}$. Conversely, any fixed point of $F$ is of this form and $\Sigma_{1}=\left\{x_{1}^{*}\right\}$ is a period 1 orbit of (1). Hence, for the stability of $\Sigma_{1}$ for (6), (7), we may analyze the stability of the corresponding fixed point of $F$. To analyze the latter, let us find the Jacobian $J_{F}$ of $F$ at $\Sigma_{1}$, i.e., $J_{F}=\left.\frac{\partial F}{\partial x}\right|_{\Sigma_{1}}$. Note that $J_{F} \in \mathbf{R}^{2 n \times 2 n}$, and let $J_{F}(i, j)$ denote its $n \times n$ blocks. These block components can be found as $J_{F}(1,1)=0, J_{F}(1,2)=I, J_{F}(2,1)=$ $-K, J_{F}(2,2)=J_{1}+K$, where $J_{1}=\left.\frac{\partial f}{\partial x}\right|_{x=x_{1}^{*}}, 0$ and $I$ are the zero and identity matrices, respectively, in appropriate dimensions. To find the characteristic polynomial $p_{1}(\lambda)=\operatorname{det}\left(\lambda I-J_{F}\right)$, let us define an auxiliary matrix $E \in \mathbf{R}^{2 n \times 2 n}$, and let $E(i, j)$ denote its $n \times n$ blocks, defined as $E(1,1)=I, E(1,2)=0$, $E(2,1)=-K, E(2,2)=\lambda I$. Note that $E$ is a lower block diagonal matrix. It can be easily verified that $R=E\left(\lambda I-J_{F}\right)$ is an upper diagonal matrix with the block components given as $R(1,1)=\lambda I, R(1,2)=$ $-I, R(2,1)=0, R(2,2)=\lambda^{2} I-\lambda\left(J_{1}+K\right)+K$. By using $\operatorname{det} R=\operatorname{det} E \operatorname{det}(\lambda I-J)$, and using the lower and upper diagonal forms of $E$ and $R$, respectively, we obtain

$p_{1}(\lambda)=\operatorname{det}\left(\lambda I-J_{F}\right)=\operatorname{det}\left(\lambda^{2} I-\lambda\left(J_{1}+K\right)+K\right)$.

We say that a polynomial is Schur stable if all of its eigenvalues are inside the unit disc of the complex plane, i.e., have magnitude less than unity. It is called unstable if at least one of its roots is outside the unit disc, and is called marginally stable if at least one of its roots is on the unit disc while the rest of the roots are inside the unit disc. Hence, the asymptotic stability of the fixed point of $F$ for (9), hence the asymptotic stability of $\Sigma_{1}$ for (6) and (7) could be analyzed by studying the Schur stability of $p_{1}(\lambda)$ given by (10). Moreover note that the exponential stability of the fixed points of $F$ is equivalent to Schur stability of $p_{1}(\lambda),[21]$. From the analysis given above we may state the following result:

Corollary 1. Let $\Sigma_{1}=\left\{x_{1}^{*}\right\}$ be a period 1 solution of (1), and consider the DFC given by (6), (7) for $T=1$. Then:

(i) $\Sigma_{1}$ is exponentially stable for (6), (7) if and only if $p_{1}(\lambda)$ given by (12) is Schur stable. This condition is only sufficient for the asymptotic stability of $\Sigma_{1}$;

(ii) if $p_{1}(\lambda)$ is unstable, then $\Sigma_{1}$ cannot be asymptotically stabilized by $D F C$;

(iii) if $p_{1}(\lambda)$ is marginally stable, then $\Sigma_{1}$ cannot be exponentially stabilized by DFC, and we cannot conclude the asymptotical stability with the analysis given above.

Proof. The proofs of these statements follow easily from the analysis given above and from standard Lyapunov stability theory, see, e.g., [21].

We proceed to the general case $T=m$. Let $\Sigma_{m}=$ $\left\{x_{1}^{*}, \ldots, x_{m}^{*}\right\}$ be the period $m$ solution of (1). Let us define the Jacobians $J_{i}$ as follows:

$J_{i}=\left.\frac{\partial f}{\partial x}\right|_{x=x_{i}^{*}}, \quad i=1, \ldots, m$.

Let us define the following variables:

$x_{j}=x(k-m-1+j), \quad j=1,2, \ldots, m+1$, 
and set $\hat{x}=\left(x_{1} \ldots x_{m+1}\right)^{T} \in \mathbf{R}^{(m+1) n}$. We can rewrite (6), (7) as

$\hat{x}(k+1)=G(\hat{x}(k))$,

where $G$ is given as

$G(\hat{x})=\left(x_{2} x_{3} \ldots x_{m+1} f\left(x_{m+1}\right)+K\left(x_{m+1}-x_{1}\right)\right)^{T}$.

It can easily be seen that the fixed points of $G$ do not correspond to $\Sigma_{m}$. To obtain such a correspondence, let us define a new map $F=G^{m}$. By a simple iteration, it can be shown that $F$ has the following form:

$F(\hat{x})=\left(Y_{1} Y_{2} \ldots Y_{m+1}\right)^{T}$,

$Y_{1}=x_{m+1}$,

$Y_{i+1}=f\left(Y_{i}\right)+K\left(Y_{i}-x_{i}\right), \quad i=1,2, \ldots, m$.

Now consider the system given by (9) with (15), (16). Next result clarifies the relation between the fixed points of $F$ and $\Sigma_{m}$.

Theorem 1. Let $\Sigma_{m}=\left\{x_{1}^{*}, \ldots, x_{m}^{*}\right\}$ be the period $m$ solution of (1). Then $\hat{x}^{*}=\left(x_{1}^{*} \ldots x_{m}^{*} x_{1}^{*}\right)^{T}$ is a fixed point of $F$. Conversely, any fixed point of $F$ has this form and the set $\Sigma_{m}$ given by (2) corresponds to a period $m$ solution of (1).

Proof. Let $\hat{x}^{*}=\left(x_{1}^{*} \ldots x_{m}^{*} x_{m+1}^{*}\right)^{T}$ be a fixed point of $F$. By using $F\left(\hat{x}^{*}\right)=\hat{x}^{*}$, we obtain $Y_{i}=x_{i}^{*}$, $i=1,2, \ldots, m+1$. This implies $x_{m+1}^{*}=x_{1}^{*}, x_{i+1}^{*}=$ $f\left(x_{i}^{*}\right), i=1,2, \ldots, m$. The result then easily follows.

Hence, to study the asymptotic stability of $\Sigma_{m}$ for (6), (7), we could study the stability of the corresponding fixed point $\hat{x}^{*}$ for (9). To analyze the latter, first we linearize $F$ at $\hat{x}^{*}$. Let us define the Jacobian of $F$ as $J_{F}=\left.\frac{\partial F}{\partial x}\right|_{\Sigma_{m}}$. Note that $J_{F}$ is an $(m+1) n \times(m+1) n$ matrix. As before, let $J(i, j)$ denote its $n \times n$ blocks, $i, j=1,2, \ldots, m+1$. From (15) clearly we have $J_{F}(i, j)=\left.\frac{\partial Y_{i}}{\partial x_{j}}\right|_{\Sigma_{m}}$. By using (16), and noting that $Y_{i}=x_{i}^{*}$ at fixed point, we obtain

$J_{F}(1, m+1)=I$,

$J_{F}(1, j)=0, \quad j=1,2, \ldots, m$,

$J_{F}(i+1, j)=\left(J_{i}+K\right) J_{F}(i, j)-\delta_{i, j} K$, where $\delta_{i, i}=1$, and $\delta_{i, j}=0$ for $i \neq j$. By using (17), (18) recursively we can easily obtain $J_{F}$. After these iterations, we find the block components of $J_{F}$ as follows: for $1 \leqslant i \leqslant m+1,1 \leqslant j \leqslant m$

$$
\begin{aligned}
& J_{F}(i, j) \\
& \quad= \begin{cases}0, & i-j<1, \\
-K, & i-j=1, \\
-\left(\prod_{l=j+1}^{i-1}\left(J_{i+j-l}+K\right)\right) K, & i-j>1\end{cases}
\end{aligned}
$$

and for $j=m+1,2 \leqslant i \leqslant m+1$ we have

$J_{F}(1, m+1)=I$,

$J_{F}(i, m+1)=\prod_{l=1}^{i-1}\left(J_{i-l}+K\right)$.

Note that the order of multiplication is important here since each term is a matrix, i.e., we have

$\prod_{l=k}^{p} D_{l}= \begin{cases}D_{k} D_{k+1} \ldots D_{p}, & p \geqslant k, \\ 0, & p<k .\end{cases}$

Now, for the stability analysis, we will find the characteristic polynomial of $F$, i.e.,

$p_{m}(\lambda)=\operatorname{det}\left(\lambda I-J_{F}\right)$.

Since all components of $J_{F}$ given by (19), (20) are matrices, to find $p_{m}(\lambda)$ is not trivial. Our approach is similar to the procedure we used for the case $m=1$. We first find a lower block diagonal matrix $E$ such that $R=E\left(\lambda I-J_{F}\right)$ is upper diagonal. Then by using $\operatorname{det} R=\operatorname{det} E \operatorname{det}\left(\lambda I-J_{F}\right)$, we can evaluate $p_{m}(\lambda)$ easily.

Let $E(i, j)$ denote $(i, j)$ th block matrix components of $E$, where $i, j=1,2, \ldots, m+1$. By utilizing the structure of $J_{F}$ given by (20), (21), after straightforward calculations we obtain a suitable matrix $E$ as follows:

$$
E(i, j)= \begin{cases}0, & i<j, \\ \lambda^{i-1} I, & i=j, \\ \lambda^{i-2} J_{F}(i, j), & j=i-1, \\ \lambda^{i-2} J_{F}(i, j) & \\ \quad+\sum_{l=1}^{i-j-1} \lambda^{i-2-l} X_{l}^{i, j}, & j<i-1 .\end{cases}
$$

$$
\begin{aligned}
X_{l}^{i, j}= & \sum_{i_{1}=1}^{i-j} \sum_{i_{2}=i_{1}+1}^{i-j} \ldots \sum_{i_{l}=i_{l-1}+1}^{i-j} J_{F}\left(i, i-i_{1}\right) \\
& \times J_{F}\left(i-i_{1}, i-i_{2}\right) \cdots J_{F}\left(i-i_{l}, j\right) .
\end{aligned}
$$


By using (19), (20), (23), (24) we see that $R$ has upper diagonal form. Similar to $E$, let $R(i, j)$ denote the block matrix components of $R$. The diagonal components of $R$ are given as

$$
\begin{aligned}
& R(i, i) \\
& = \begin{cases}\lambda^{i} I, & i \leqslant m, \\
\lambda^{m+1} I-\lambda^{m} J_{F}(m+1, m+1) & \\
\quad-\sum_{l=1}^{m} \lambda^{m-l} X_{l}, & i=m+1 .\end{cases} \\
& X_{l}=\sum_{i_{1}=1}^{m} \sum_{i_{2}=i_{1}+1}^{m} \ldots \sum_{i_{l}=i_{l-1}+1}^{m} J_{F}\left(m+1, i-i_{1}\right) \\
& \times J_{F}\left(i-i_{1}, i-i_{2}\right) \cdots J_{F}\left(i-i_{l}, m+1\right) .
\end{aligned}
$$

From (23)-(26) we obtain $\operatorname{det} E=\lambda^{N}$ and $\operatorname{det} R=$ $\lambda^{N} \operatorname{det} R(m+1, m+1)$, where $N=m(m+1) n / 2$. By using these, we obtain

$p_{m}(\lambda)=\operatorname{det}\left(\lambda I-J_{F}\right)=\operatorname{det} R(m+1, m+1)$,

where $R(m+1, m+1)$ is given by (25), (26). By using (19), (20) in (25), (26), equivalently we obtain the following:

$p_{m}(\lambda)=\operatorname{det}\left(\sum_{l=0}^{m+1} \lambda^{l} P_{l}\right)$,

where the matrices $P_{l}$ are given as

$$
\begin{aligned}
P_{m+1}= & I, \quad P_{m}=-\prod_{i=0}^{m-1}\left(J_{m-i}+K\right) \\
P_{0}=- & (-1)^{m} K^{m} \\
P_{m-l}= & -(-1)^{l} \\
& \times \sum_{i_{1}=1}^{m} \sum_{i_{2}=i_{1}+1}^{m} \ldots \sum_{i_{l}=i_{l-1}+1}^{m} \prod_{i=0}^{m-1}\left(D_{m-i}+K\right), \\
1<l< & l<,
\end{aligned}
$$

and the matrices $D_{k}$ are defined as

$D_{k}= \begin{cases}J_{k}, & k \neq\left\{i_{1}, i_{2}, \ldots, i_{l}\right\}, \\ 0, & k \in\left\{i_{1}, i_{2}, \ldots, i_{l}\right\} .\end{cases}$

Remark 1. The formulas given by (28)-(31) may look complicated, but their interpretations are rather simple. Let us denote $P_{m-l}=-(-1)^{l} \hat{P}_{m-l}$. Now consider the ordered set $\mathcal{I}_{m}=\{m, m-1, \ldots, 1\}$ and an arbitrary $l$ selection of indices from $\mathcal{I}_{m}$ as $\sigma_{l}=\left\{i_{1}, i_{2}, \ldots, i_{l}\right\}$ preserving the order, i.e., $i_{1}>$ $i_{2}>\cdots>i_{l}$. A typical term in $\hat{P}_{m-l}$ is of the form $\Gamma_{m} \Gamma_{m-1} \ldots \Gamma_{1}$, where $\Gamma_{i}=K$ if $i \in \sigma_{l}$, and $\Gamma_{i}=$ $J_{i}+K$ if otherwise, see (30), (31). Then, $\hat{P}_{m-l}$ is the sum of all possible such terms. For the case $m=1, p_{1}(\lambda)$ given above is the same as (10). As an example, for the case $m=2$, the matrices given by (28)-(31) becomes $P_{2}=-\left(J_{2}+K\right)\left(J_{1}+K\right)$, $P_{1}=\left(J_{2}+K\right) K+K\left(J_{1}+K\right), P_{0}=-K^{2}$. For the case $m=3$, these matrices are given as $P_{3}=$ $-\left(J_{3}+K\right)\left(J_{2}+K\right)\left(J_{1}+K\right), P_{2}=\left(J_{3}+K\right)\left(J_{2}+\right.$ $K) K+\left(J_{3}+K\right) K\left(J_{1}+K\right)+K\left(J_{2}+K\right)\left(J_{1}+K\right)$, $P_{1}=-\left(J_{3}+K\right) K^{2}-K\left(J_{2}+K\right) K-K^{2}\left(J_{1}+K\right)$, $P_{0}=K^{3}$.

The characteristic polynomial $p_{m}(\lambda)$ given by (28) is related to the periodic orbit $\Sigma_{m}$ given by (2). Note that in constructing $p_{m}(\lambda)$, the order of the periodic points is important, see (29)-(31). On the other hand, any circular permutation of the periodic points in $\Sigma_{m}$ corresponds to the same periodic orbit, and such permutations may correspond to different characteristic polynomials due to the fact that both Jacobians and the gain $K$ are matrices whose products may not commute. To take this point into account, let us define any circular permutation of $\Sigma_{m}$ as follows:

$\Sigma_{m}^{j}=\left\{x_{j}^{*}, x_{j+1}^{*}, \ldots, x_{j+m-1}^{*}\right\}, \quad 1 \leqslant j \leqslant m$,

where we used circular notation, i.e., $x_{i}^{*}=x_{j}^{*}$ if $i=$ $j(\bmod m)$. Let $p_{m}^{j}(\lambda)$ denote the characteristic polynomial corresponding to $\Sigma_{m}^{j}$. Note that with this notation we have $\Sigma_{m}^{1}=\Sigma_{m}$. We now state the following result.

Theorem 2. Let $\Sigma_{m}$ given by (2) be a period $m$ orbit of (1). Let $\Sigma_{m}^{j}$ given by (32) be any circular permutation of $\Sigma_{m}$ and let $p_{m}^{j}(\lambda)$ be the corresponding characteristic polynomial given by (28)-(31). The DFC scheme given by (6), (7) is:

(i) locally exponentially stable if and only if at least one of the polynomials $p_{m}^{j}(\lambda)$ is Schur stable;

(ii) unstable if all of the polynomials $p_{m}^{j}(\lambda)$ are unstable;

(iii) if all $p_{m}^{j}(\lambda)$ are marginally stable, then $\Sigma_{m}$ cannot be exponentially stabilized by DFC, and we 
cannot conclude the asymptotical stability with the analysis given above.

Proof. Note that $\Sigma_{m}$ and $\Sigma_{m}^{j}$ correspond to the same periodic orbit. Hence, stabilization of any $\Sigma_{m}^{j}$ is the same as the stabilization of the periodic orbit in question. Also note that exponential stability is equivalent to the stability of linearization, see, e.g., [21]. The proof of the theorem then follow easily from the analysis given above and from standard Lyapunov stability theory, see, e.g., [21].

Remark 2. Note that although $\Sigma_{m}^{j}$ given by (32) corresponds to the same periodic orbit, the related characteristic polynomials $p_{m}^{j}(\lambda)$ may be different for $n>1$, see (28)-(31). However, for the case $n=1$, these polynomials are identical, since in this case both the Jacobians and the gain are scalars, which necessarily commute. We also note that for the case $n=1$, the polynomial $p_{m}(\lambda)$ given by (28)-(31) is the same as given in [13].

Let us consider $\Sigma_{m}^{j}$ given by (32), and let us define the associated product of Jacobians as

$J_{m}^{j}=J_{j} J_{j+1} \cdots J_{j+m-1}, \quad 1 \leqslant j \leqslant m$,

where we used circular notation, i.e., $J_{j}=J_{i}$ if $i=$ $j(\bmod m)$.

Remark 3. Note that although $J_{m}^{i} \neq J_{m}^{j}$ for $i \neq j$ in general, the eigenvalues of $J_{m}^{j}$ and $J_{m}^{i}$ are exactly the same since both correspond to the same periodic orbit, see, e.g., [22].

The next result shows the well-known limitation of the DFC scheme, which is known as "odd number limitation", see, e.g., [11].

Theorem 3. Let $\Sigma_{m}$ given by (2) be a period $m$ orbit of (1). Let $\Sigma_{m}^{j}$ given by (32) be any circular permutation of $\Sigma_{m}$. Consider the product of Jacobians $J_{m}^{j}$ given by (33). If any (hence all) of $J_{m}^{j}$ has an odd number of real eigenvalues greater than 1, then DFC scheme given by (6), (7) cannot be asymptotically stable.

Proof. Note that the eigenvalues of $\Sigma_{m}^{j}$ are the same for $j=1,2, \ldots, m$, see Remark 3. A well-known necessary condition for the Schur stability of any polynomial $p(\lambda)$ is that $p(1)>0$ should hold, see, e.g., [23]. Now consider the polynomial $p_{m}(\lambda)$ given by (28)-(31), which corresponds to $p_{m}^{1}$. Here we have $p_{m}(1)=\operatorname{det}\left(\sum_{l=1}^{m+1} P_{l}\right)$. By using (29)-(31), after straightforward calculations it can be shown that we have $p_{m}(1)=\operatorname{det}\left(I-J_{m}^{1}\right)$, i.e., the contribution of the terms containing the gain $K$ cancels. By using Jordan canonical form, it can be shown easily that $p_{m}(1)>0$ if and only if the number of real eigenvalues of $J_{m}^{1}$ greater than 1 is even. Therefore, if this number is odd, then DFC cannot be stable.

Remark 4. The condition given in Theorem 3 can be interpreted as an inherent limitation of DFC. This limitation is known as odd number limitation, and for alternative proofs see, e.g., $[11,12,20]$.

Note that the DFC scheme achieves only local stabilization. Hence, for implementation it should be applied when the solutions are sufficiently close to the periodic orbit $\Sigma_{m}$. Following [13], to achieve this aim we may use the following implementation for (7):

$$
\begin{aligned}
& u(k)=\epsilon(k)(x(k)-x(k-m)), \\
& \epsilon(k)= \begin{cases}K, & d(k) \leqslant \epsilon_{m}, \\
0, & d(k)>\epsilon_{m},\end{cases}
\end{aligned}
$$

where $d(k)$ is a distance measure for the domain of attraction of $\Sigma_{m}$, and $\epsilon_{m}$ is a measure of the domain of attraction of $\Sigma_{m}$. Now let us consider the selection of $d(k)$ in (35). The distance measure given by (3), (4) is not suitable from implementation point of view, since $m$ iterates of (1) starting from $x(k)$ are compared with $\Sigma_{m}$, whereas to compute $u(k)$ we could only use the past iterates. For this reason, instead of (3), we modify $d_{k}(j)$ for the simulations as follows $(j=1,2, \ldots, m)$ :

$d_{k}(j)=\sqrt{\sum_{i=0}^{m-1}\left\|x(k-m+1+i)-x_{i+j}^{*}\right\|^{2}}$.

We could choose either $d(k)=d_{k}(j)$ or as $d(k)=$ $d\left(x(k), \Sigma_{m}\right)=\min \left\{d_{k}(1), \ldots, d_{k}(m)\right\}$ where $d_{k}(j)$ is given as in (36). We will use the latter choice for the implementation of (7) in our simulations.

Remark 5. Let $\Sigma_{m}$ given by (2) be a period $m$ orbit of (1) and let $p_{m}^{j}(\lambda)$ be the corresponding characteristic 
polynomial given by (28)-(31). Although the Theorem 2 gives necessary and sufficient conditions for the exponential stability of $\Sigma_{m}$ for the DFC scheme given by (6), (7), it is more suitable to decide whether a given $K$ achieves this aim. If a stabilizing $K$, if it exists, is to be found than the best way is to use a numerical search by keeping the gain components as variables and testing the stability of polynomials given by (28)(31) by using numerical methods. For $n=1$ and small $m$, some analytical results may be obtained, see, e.g., [13]. However, as $n$ and/or $m$ increases, finding analytical results become increasingly difficult and one has to resort to numerical methods to find a suitable $K$. Note that $K$ is and $n \times n$ matrix, and computational complexity of finding a suitable $K$ increases possibly exponentially with $n$. Therefore, to keep the computational complexity low, we used the cases $n=2$ in our simulations.

\section{Simulation results}

We first considered the well-known Hénon map given as

$f(w)=\left(\begin{array}{c}1+y-1.4 x^{2} \\ 0.3 x\end{array}\right)$,

where $w=(x y)^{T} \in \mathbf{R}^{2}$. Note that instead of the notation $x$ in (1), (6) and (7) to denote the state variable, we use $w$ here since it is customary to use the labels $x$ and $y$ for the variables in Hénon map. This map has period 2 solution characterized by the set $\Sigma_{2}=\left\{w_{1}^{*}, w_{2}^{*}\right\}$ where

$w_{1}^{*}=\left(\begin{array}{c}0.975800051 \\ -0.142740015\end{array}\right)$,

$w_{2}^{*}=\left(\begin{array}{c}-0.475800051 \\ 0.292740015\end{array}\right)$.

The Jacobians $J_{1}$ and $J_{2}$ can be computed as

$$
\begin{aligned}
& J_{1}=\left.\frac{\partial f}{\partial w}\right|_{w=w_{1}^{*}}=\left(\begin{array}{cc}
-2.7322 & 1 \\
0.3 & 0
\end{array}\right), \\
& J_{2}=\left.\frac{\partial f}{\partial w}\right|_{w=w_{2}^{*}}=\left(\begin{array}{cc}
1.3322 & 1 \\
0.3 & 0
\end{array}\right) .
\end{aligned}
$$

The characteristic polynomial is given by (28)-(31) is computed as

$$
\begin{aligned}
p_{2}(\lambda)=\operatorname{det} & \left(\lambda^{3} I-\lambda^{2}\left(J_{2}+K\right)\left(J_{1}+K\right)\right. \\
& \left.+\lambda\left(\left(J_{2}+K\right) K+K\left(J_{1}+K\right)\right)-K^{2}\right) .
\end{aligned}
$$

After straightforward calculations, we find some stabilizing gains. For simulations we choose the following gain

$K=\left(\begin{array}{cc}-0.4 & 0 \\ 0.2 & -0.2\end{array}\right)$.

We simulated (6), (34) and (35) for the map given by (37) with the gain (41) and $\epsilon_{m}=0.1$. Initial conditions are chosen as $x(1)=0.5, y(1)=0$. The results of the simulation are shown in Fig. 1. In Fig. 1(a), we show $d\left(w(k), \Sigma_{2}\right)$ versus $k$, and as can be seen the decay is exponential for $k \geqslant 300$. The $x(k)$ versus $y(k)$ plot in Fig. 1(b) is plotted for $k \geqslant 400$. As can be seen from these figures, the solutions converge to the period 2 orbit characterized by $\Sigma_{2}$. Finally, the required input components $u_{1}(k)$ and $u_{2}(k)$, where $u(k)=\left(u_{1}(k) u_{2}(k)\right)^{T}$, are shown in Fig. 1(c) and (d), respectively. As can be seen from these figures, $u(k) \rightarrow 0$ as $k \rightarrow \infty$.

In the second simulation, we consider the wellknown Ikeda Laser map given below

$f(w)=\left(\begin{array}{c}r+c_{2}(x \cos \tau-y \sin \tau) \\ c_{2}(x \sin \tau+y \cos \tau)\end{array}\right)$,

where $w=(x y)^{T} \in \mathbf{R}^{2}$, and $\tau=c_{1}-c_{3} /\left(1+x^{2}+\right.$ $\left.y^{2}\right)$. For the parameter values, we choose $r=1, c_{1}=$ $0.4, c_{2}=0.9, c_{3}=6$, for which this system has a well known chaotic attractor, see [22]. This map has period 2 solution characterized by the set $\Sigma_{2}=\left\{w_{1}^{*}, w_{2}^{*}\right\}$ where

$$
\begin{aligned}
& w_{1}^{*}=\left(\begin{array}{c}
0.621604323 \\
0.509837250
\end{array}\right), \\
& w_{2}^{*}=\left(\begin{array}{c}
0.605933647 \\
-0.608369928
\end{array}\right) .
\end{aligned}
$$

The related Jacobians and the characteristic polynomial can be found by using (42), (39) and (40). After straightforward calculations, we find some stabilizing gains. For simulations we choose the following gain which yielded smallest eigenvalues for $p_{2}(\lambda)$ :

$K=\left(\begin{array}{cc}0.6 & -0.45 \\ 0.8 & -0.5\end{array}\right)$. 


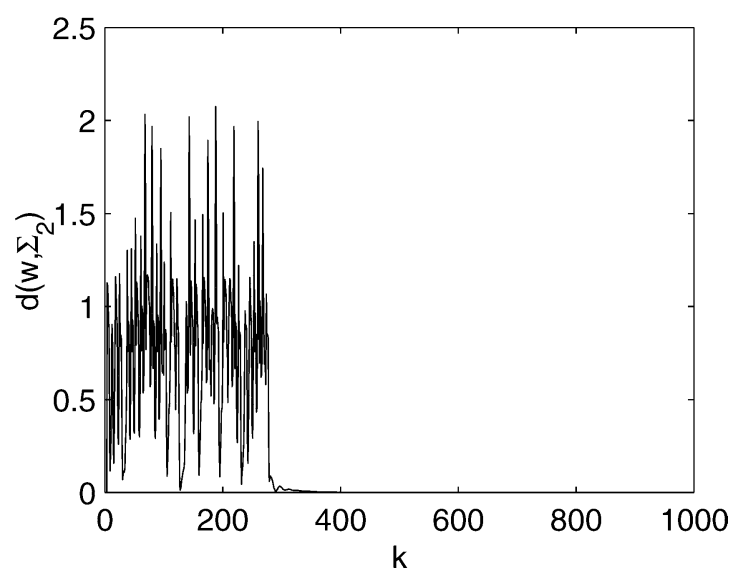

(a)

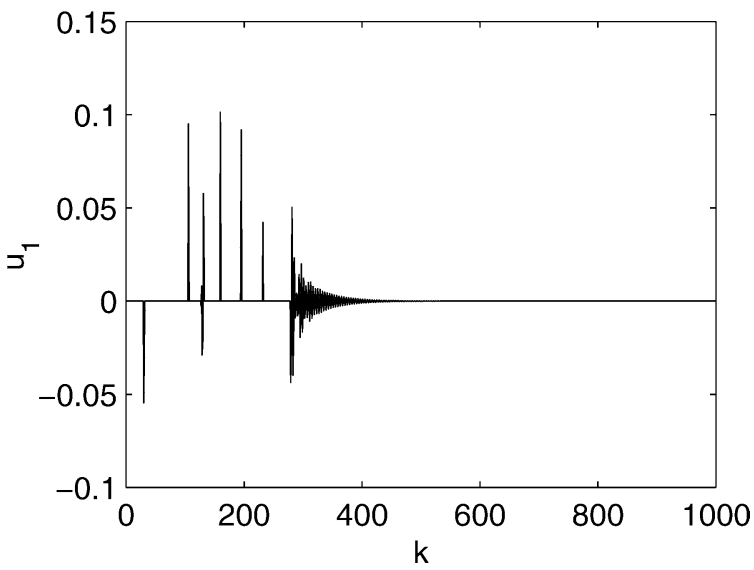

(c)

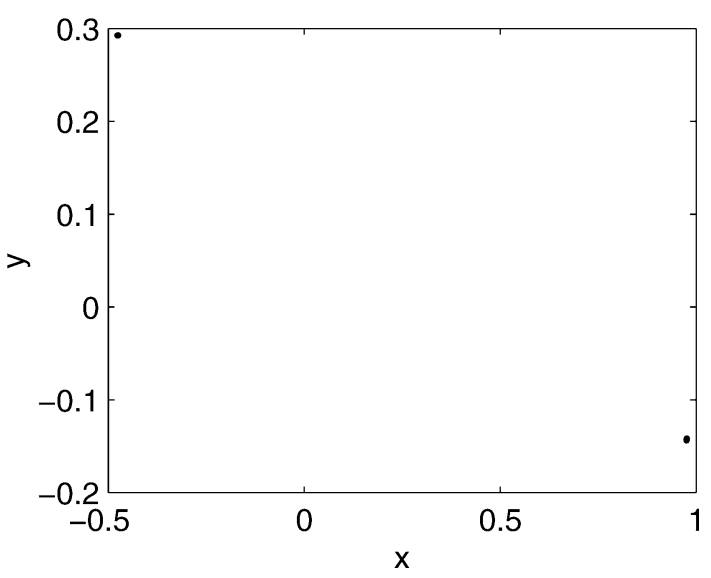

(b)

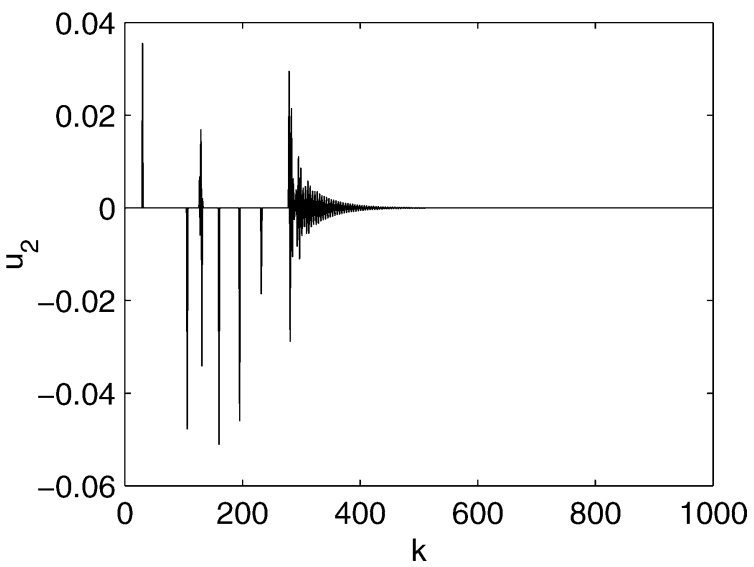

(d)

Fig. 1. DFC applied to Hénon map: (a) $d\left(w(k), \Sigma_{2}\right)$ vs. $k$; (b) $x(k)$ vs. $y(k)$ for $k \geqslant 400$; (c) $u_{1}(k)$ vs. $k$; (d) $u_{2}(k)$ vs. $k$.

We simulated (6), (34) and (35) for the map given by (42) with the gain (44) and $\epsilon_{m}=0.1$. Initial conditions are chosen as $x(1)=0.9, y(1)=0.3$. The results of the simulation are shown in Fig. 2. In Fig. 2(a), we show $d\left(w(k), \Sigma_{2}\right)$ versus $k$, and as can be seen the decay is exponential for $k \geqslant 200$. The $x(k)$ versus $y(k)$ plot in Fig. 2(b) is plotted for $k \geqslant 300$. As can be seen from these figures, the solutions converge to the period 2 orbit characterized by $\Sigma_{2}$. Finally, the required input components $u_{1}(k)$ and $u_{2}(k)$, where $u(k)=\left(u_{1}(k) u_{2}(k)\right)^{T}$, are shown in Fig. 2(c) and (d), respectively. As can be seen from these figures, $u(k) \rightarrow 0$ as $k \rightarrow \infty$.

In the last two simulations, we considered the coupled map lattices, which exhibit various interesting dynamical behaviours, see, e.g., [24]. We will use the fol- lowing one-dimensional unidirectionally coupled lattices:

$x_{k+1}(i)=g\left(x_{k}(i)\right)+\epsilon\left(g\left(x_{k}(i-1)\right)-\left(x_{k}(i)\right)\right)$,

where $g: \mathbf{R} \rightarrow \mathbf{R}, i=1,2, \ldots, L$ denotes the lattice sites, $L$ indicates the system size, $k=0,1, \ldots$ indicates the discrete time index, and as is usual we apply periodic boundary site conditions, i.e., $x_{k}(i+L)=$ $x_{k}(i)$, and $\epsilon$ is a coupling constant. For an application of this model to coupled diode resonators, see, e.g., [25]. We will use this system for $L=2$ with the wellknown logistic and tent maps. The reason for choosing $L$ low is basically because of computational difficulties in finding an appropriate gain, see the Remark 5. Note that in this case, $K$ will be a $L \times L$ matrix, and 


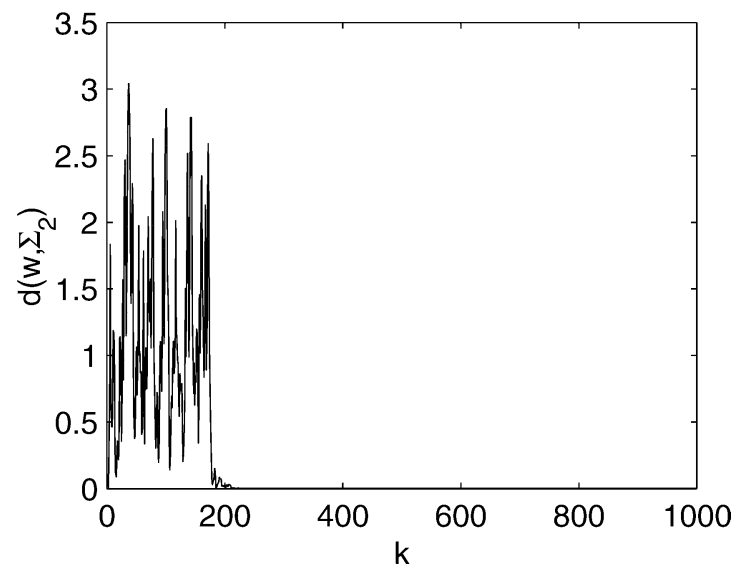

(a)

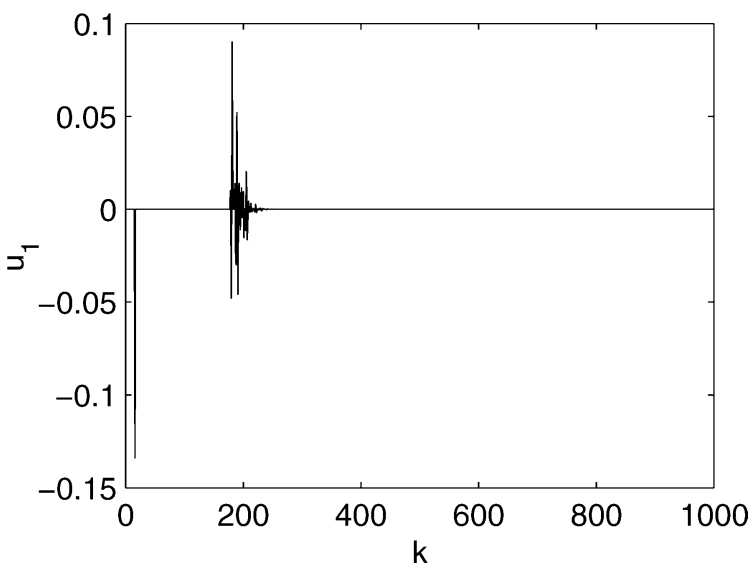

(c)

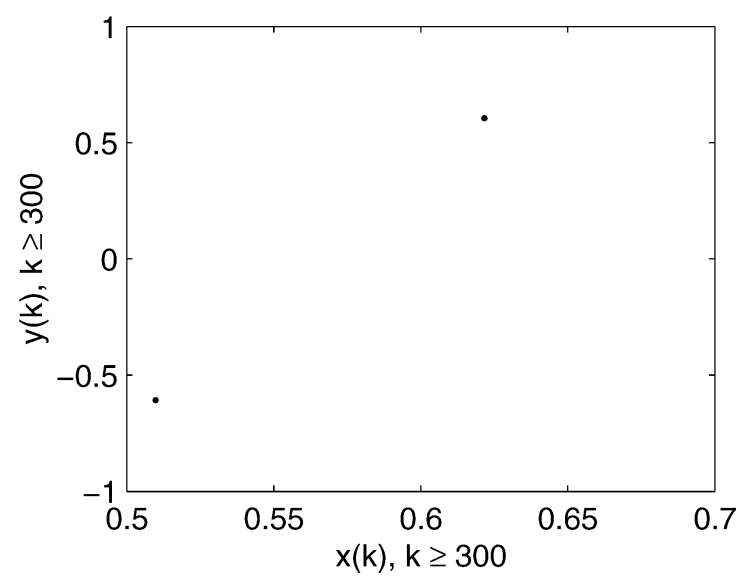

(b)

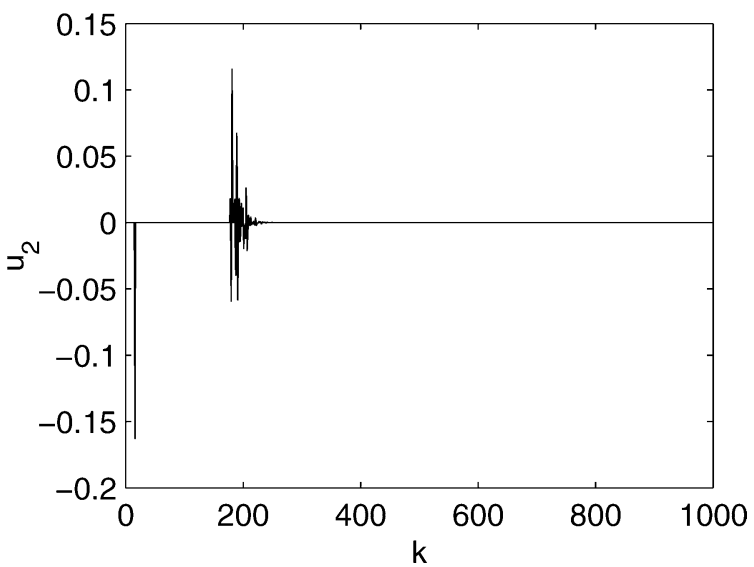

(d)

Fig. 2. DFC applied to Ikeda laser map: (a) $d\left(w(k), \Sigma_{2}\right)$ vs. $k$; (b) $x(k)$ vs. $y(k)$ for $k \geqslant 300$; (c) $u_{1}(k)$ vs. $k$; (d) $u_{2}(k)$ vs. $k$.

finding a stabilizing gain using the determinant given by (28)-(31) will become increasingly difficult as we increase $L$. Most possibly, the required calculations will increase exponentially as we increase $L$.

For $L=2$, and for a given map $g(x)$, the dynamics is given by the following map:

$f(w)=\left(\begin{array}{l}g(x)+\epsilon(g(y)-g(x)) \\ g(y)+\epsilon(g(x)-g(y))\end{array}\right)$,

where $w=(x y)^{T} \in \mathbf{R}^{2}$.

First we will consider the logistic map given as $g(x)=r x(1-x)$. For $r=3.87$ and $\epsilon=0.99$, the map given by (46) has a period 3 orbit characterized by the set $\Sigma_{3}=\left\{w_{1}^{*}, w_{2}^{*}, w_{3}^{*}\right\}$ where

$w_{1}^{*}=\left(\begin{array}{c}0.4450407828 \\ 0.526709116\end{array}\right)$,

$$
\begin{aligned}
& w_{2}^{*}=\left(\begin{array}{l}
0.964649945 \\
0.955899891
\end{array}\right), \\
& w_{3}^{*}=\left(\begin{array}{l}
0.162829246 \\
0.132280381
\end{array}\right) .
\end{aligned}
$$

The Jacobians $J_{i}$ can easily be calculated by using (46). The characteristic polynomial given by (28)-(31) can be computed as $p_{3}(\lambda)=\operatorname{det}\left(\lambda^{4} I+P_{3} \lambda^{3}+P_{2} \lambda^{2}+\right.$ $\left.P_{1} \lambda+P_{0}\right)$ with $P_{3}=-\left(J_{3}+K\right)\left(J_{2}+K\right)\left(J_{1}+K\right)$, $P_{2}=\left(J_{3}+K\right)\left(J_{2}+K\right) K+\left(J_{3}+K\right) K\left(J_{1}+K\right)+$ $K\left(J_{2}+K\right)\left(J_{1}+K\right), P_{1}=-\left(J_{3}+K\right) K^{2}-K\left(J_{2}+\right.$ $K) K-K^{2}\left(J_{1}+K\right), P_{0}=K^{3}$. After an extensive search, we find the following stabilizing gain yielding smallest roots for $p_{3}(\lambda)$ :

$$
K=\left(\begin{array}{cc}
0.1745 & 0.0260 \\
-0.1255 & 0.0255
\end{array}\right) \text {. }
$$




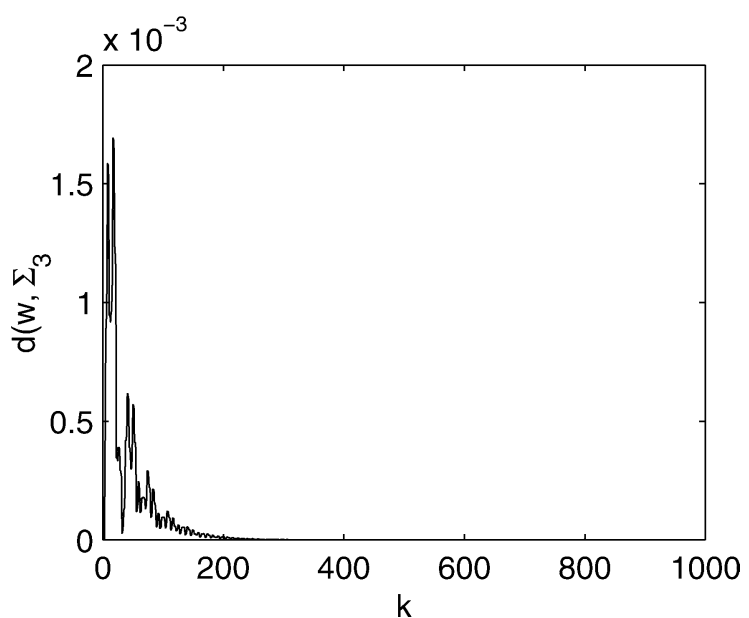

(a)

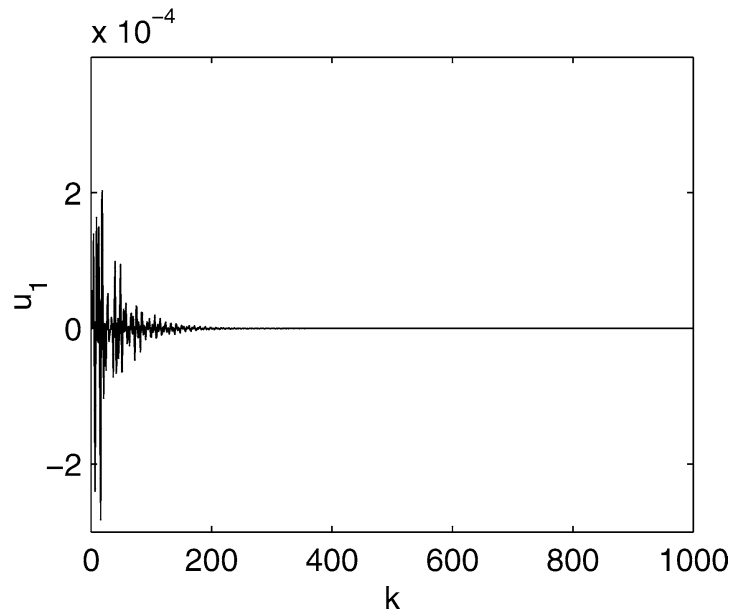

(c)

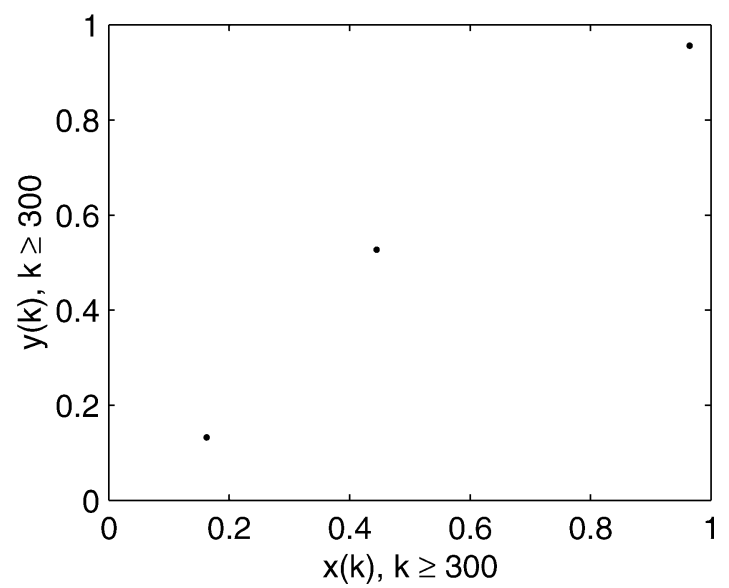

(b)

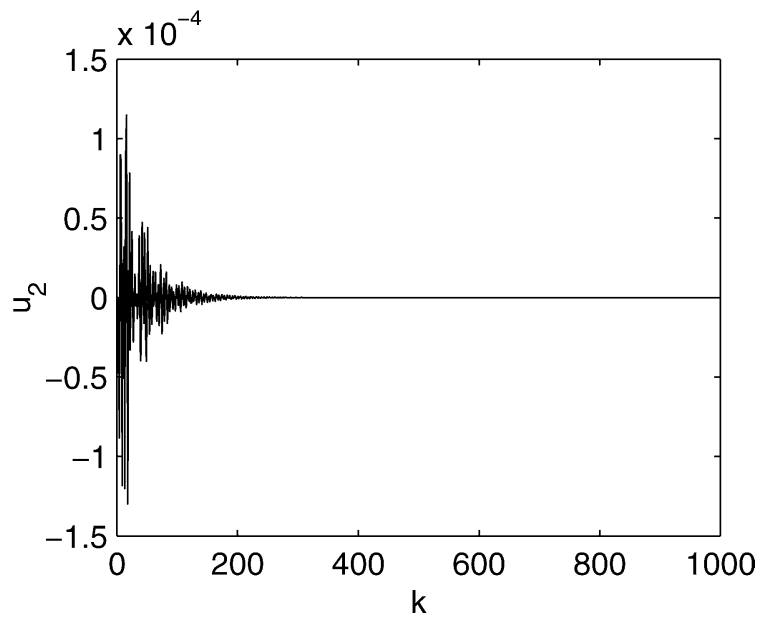

(d)

Fig. 3. DFC applied to coupled logistic map lattice: (a) $d\left(w(k), \Sigma_{3}\right)$ vs. $k$; (b) $x(k)$ vs. $y(k)$ for $k \geqslant 300$; (c) $u_{1}(k)$ vs. $k$; (d) $u_{2}(k)$ vs. $k$.

We simulated (6), (34) and (35) for the map given by (46) and the logistic map, with the gain (48) and $\epsilon_{m}=$ 0.1 . Initial conditions are chosen as $x(1)=0.445$, $y(1)=0.527$. The results of the simulation are shown in Fig. 3. In Fig. 3(a), we show $d\left(w(k), \Sigma_{3}\right)$ versus $k$. The $x(k)$ versus $y(k)$ plot in Fig. 3(b) is plotted for $k \geqslant 300$. As can be seen from these figures, the solutions converge to the period 3 orbit characterized by $\Sigma_{3}$. Finally, the required input components $u_{1}(k)$ and $u_{2}(k)$, where $u(k)=\left(u_{1}(k) u_{2}(k)\right)^{T}$, are shown in Fig. 3(c) and (d), respectively. As can be seen from these figures, $u(k) \rightarrow 0$ as $k \rightarrow \infty$.

For the last set of simulations, we used the tent map given as $g(x)=m x$ for $x \leqslant 0.5$, and $g(x)=m-m x$ for $0.5<x \leqslant 1$ in (46). For $m=1.4$ and $\epsilon=0.1$, this map has period 2 solution characterized by the set $\Sigma_{2}=\left\{w_{1}^{*}, w_{2}^{*}\right\}$ where

$$
\begin{aligned}
& w_{1}^{*}=\left(\begin{array}{l}
0.490654205 \\
0.665109034
\end{array}\right), \\
& w_{2}^{*}=\left(\begin{array}{l}
0.665109034 \\
0.490654205
\end{array}\right) .
\end{aligned}
$$

The related Jacobians and the characteristic polynomial can be found by using (46), (39) and (40). After straightforward calculations, we find some stabilizing gains. For simulations we choose the following gain 


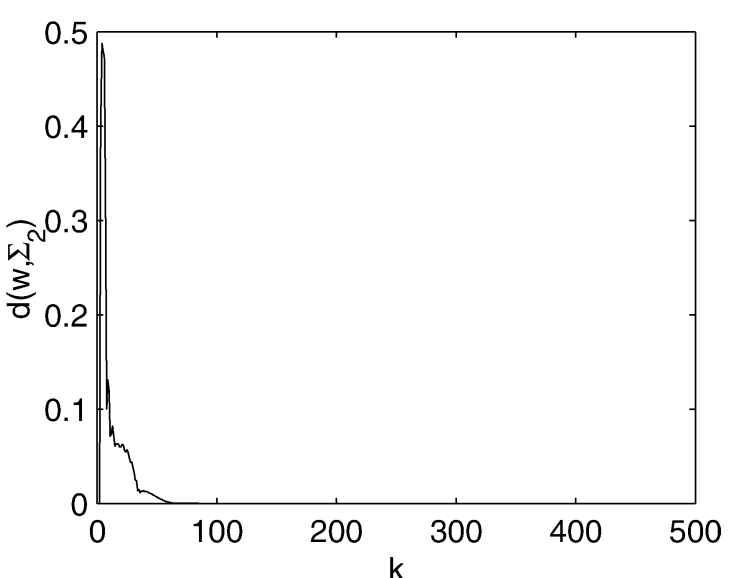

(a)

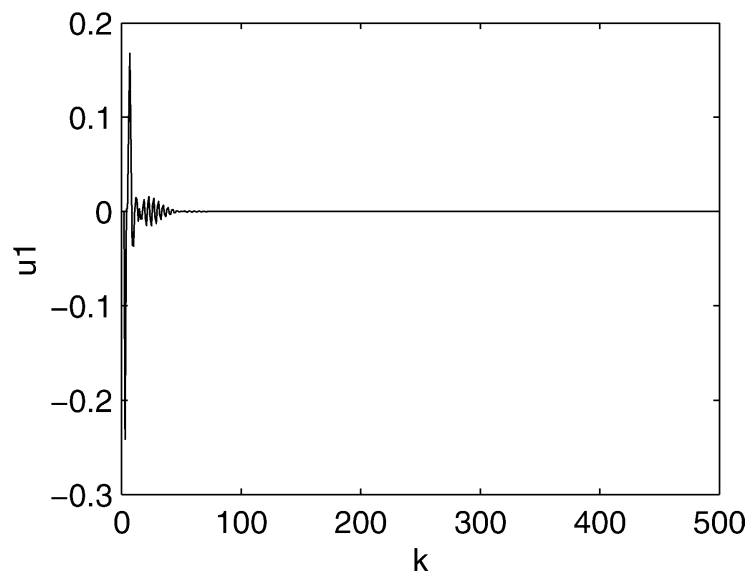

(c)

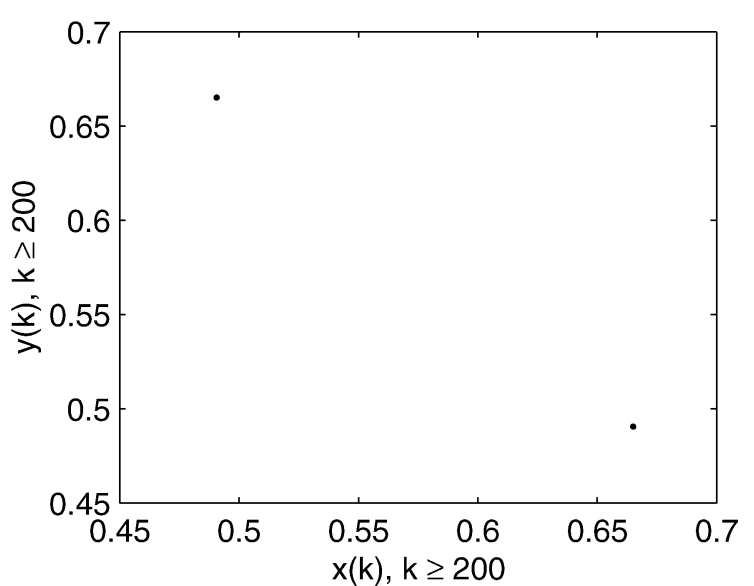

(b)

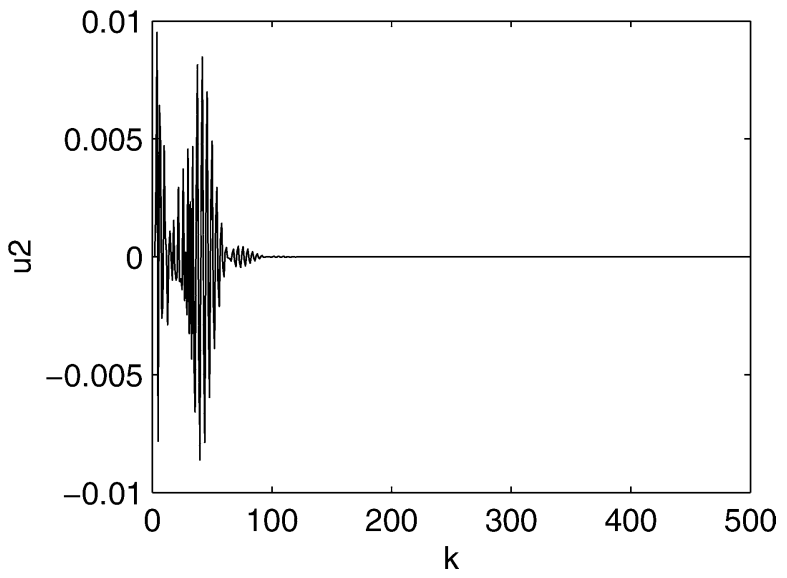

(d)

Fig. 4. DFC applied to coupled tent map lattice: (a) $d\left(w(k), \Sigma_{2}\right)$ vs. $k$; (b) $x(k)$ vs. $y(k)$ for $k \geqslant 200$; (c) $u_{1}(k)$ vs. $k$; (d) $u_{2}(k)$ vs. $k$.

which yielded smallest eigenvalues for $p_{2}(\lambda)$ :

$K=\left(\begin{array}{cc}0.4 & 0 \\ 0 & 0.4\end{array}\right)$

We simulated (6), (34) and (35) for the map given by (46) with the gain (44) and $\epsilon_{m}=0.1$ for the tent map. Initial conditions are chosen as $x(1)=0.9, y(1)=$ 0.55 . The results of the simulation are shown in Fig. 4. In Fig. 4(a), we show $d\left(w(k), \Sigma_{2}\right)$ versus $k$, and as can be seen the decay is exponential. The $x(k)$ versus $y(k)$ plot in Fig. 4(b) is plotted for $k \geqslant 200$. As can be seen from these figures, the solutions converge to the period 2 orbit characterized by $\Sigma_{2}$. Finally, the required input components $u_{1}(k)$ and $u_{2}(k)$, where $u(k)=\left(u_{1}(k) u_{2}(k)\right)^{T}$, are shown in Fig. 4(c) and (d), respectively. As can be seen from these figures, $u(k) \rightarrow 0$ as $k \rightarrow \infty$.

\section{Conclusion}

In this Letter we analyzed the stability of DFC scheme for (multi-dimensional) discrete time chaotic systems. We adopted the technique used in [13] for one-dimensional systems. We first constructed a map whose fixed points correspond to the periodic orbits of the uncontrolled chaotic system. Then we analyzed the stability of the constructed map around the fixed point corresponding to the related periodic orbit. For the stability analysis we first linearize this map around the fixed point and find the characteristic polynomial 
of the related Jacobian matrix. We give a form of this polynomial which is related to the Jacobians of the original chaotic map at the periodic orbit points and the DFC gain matrix. Then the stability of the DFC scheme can be determined by checking the Schur stability of the associated characteristic polynomial. We also presented some simulation results.

Note that, for a given $\Sigma_{m}$ and $K$, Theorem 2 can easily be used whether the given $K$ achieves stabilization. On the other hand, if a stabilizing $K$ is to be found, then by using the components of $K$ as variables, one may try to find such a $K$ by using Theorem 2 and a numerical search. The computational complexity of this search obviously increases as $n$ and/or $m$ increases. Therefore, by using Theorem 2, to develop some simple techniques which may yield a stabilizing gain, if it exists, seems to be an open problem. However, this point requires further research.

\section{References}

[1] E. Ott, C. Grebogi, J.A. Yorke, Phys. Rev. Lett. 64 (1990) 1196.

[2] S. Boccaletti, C. Grebogi, Y.C. Lai, H. Mancini, D. Maza, Phys. Rep. 329 (2000) 103.

[3] G. Chen, X. Dong, From Chaos to Order: Methodologies, Perspectives and Applications, World Scientific, Singapore, 1999.

[4] K. Pyragas, Phys. Lett. A. 170 (1992) 421.
[5] K. Pyragas, A. Tamaševičius, Phys. Lett. A 180 (1993) 99.

[6] W. Just, et al., Phys. Rev. Lett. 78 (1997) 203.

[7] S. Bielawski, D. Derozier, P. Glorieux, Phys. Rev. E 49 (1994) R971.

[8] T. Hikihara, K. Kawagishi, Phys. Lett. A 211 (1996) 29.

[9] K. Konishi, H. Kokame, K. Hirata, Phys. Rev. E 60 (1999) 4000.

[10] K. Pyragas, Phys. Rev. Lett. 86 (2001) 2265.

[11] T. Ushio, IEEE Trans. Circuits Systems I, Fund. Theory Appl. 43 (1996) 815.

[12] H. Nakajima, Phys. Lett. A 232 (1997) 207.

[13] Ö. Morgül, Phys. Lett. A 314 (2003) 278.

[14] J.E.S. Socolar, D.W. Sukow, D.J. Gauthier, Phys. Rev. E 50 (1994) 3245.

[15] A. Kittel, J. Parisi, K. Pyragas, Phys. Lett. A 198 (1995) 433.

[16] K. Pyragas, Phys. Lett. A 206 (1995) 323.

[17] M.E. Bleich, J.E.S. Socolar, Phys. Lett. A 210 (1996) 87.

[18] H.G. Schuster, M.B. Stemmler, Phys. Rev. E 56 (1997) 6410.

[19] H. Nakajima, Y. Ueda, Phys. Rev. E 58 (1998) 1757.

[20] T. Hino, S. Yamamoto, T. Ushio, Int. J. Bifur. Chaos 12 (2002) 439.

[21] H.K. Khalil, Nonlinear Systems, third ed., Prentice-Hall, Upper Saddle River, 2002.

[22] K.T. Alligood, T.D. Sauer, J.A. Yorke, Chaos, An Introduction to Dynamical Systems, Springer-Verlag, New York, 1997.

[23] S.N. Elaydi, An Introduction to Difference Equations, Springer-Verlag, New York, 1996.

[24] K. Kaneko (Ed.), Theory and Applications of Coupled Map Lattices, Wiley, Chichester, 1993.

[25] G.A. Johnson, M. Löcher, E.R. Hunt, Phys. Rev. E. 51 (1995) 1625. 